\title{
Scattering for Infinite Dimensional Port Hamiltonian Systems
}

\author{
Alessandro Macchelli ${ }^{1}$, Stefano Stramigioli ${ }^{2}$, Arjan van der Schaft ${ }^{3}$, Claudio Melchiorri ${ }^{1}$
}

\begin{abstract}
In this paper, an introduction to scattering for infinite dimensional systems within the framework of port Hamiltonian systems is presented. The classical results on wave propagation can be extended to generic power propagation phenomena, for example to fluid dynamics or flexible structures. The key-point is the generalization of the concept of impedance to other domains than the electromagnetic one.
\end{abstract}

Keywords: infinite dimensional port Hamiltonian systems, scattering, modeling

\section{Introduction}

Scattering is a well known phenomena in physics and in network and communication theory: when a wave propagating in a material medium encounters discontinuities, its properties (direction, frequency or polarization) are changed, in strict relation to the intrinsic characteristics of the material through which the wave is propagating, [1]

The amount of power exchanged during the interaction among physical systems can be calculated by means of some operations on the so-called power variables (e.g. currents and voltages, forces and velocities) defined on the ports of the systems themselves. It is on these ports that the interaction takes place. In power propagation, once an orientation is fixed for each port, it is possible to locate two distinct power flows, the incoming and the outgoing one, by means of the scattering decomposition, an operation defined on the space of power variables. In $[2,3]$, a geometric approach to scattering is presented in the case of finite dimensional systems, where the space of energy variables is a finite dimensional vector space.

In the framework of infinite dimensional port Hamiltonian system [4], the space of power variables is given by an infinite dimensional space. In this paper, it will be pointed out that it is still possible to implement a scattering decomposition that is the natural generalization

\footnotetext{
${ }^{1}$ DEIS, University of Bologna (Italy), email: \{amacchelli, cmelchiorri\}odeis.unibo.it

${ }^{2}$ Elektrotechniek, Drebbel Institute, Universiteit Twente (Netherlands), email: S.Stramigioli@ieee.org

${ }^{3}$ Toegepaste Wiskunde, Universiteit Twente (Netherlands), email: a.j.vanderschaft math. utwente.nl
}

of the finite dimensional one. This result is presented in Sec. 2, while in Sec. 3 an application to Maxwell's equations is discussed. Finally, Sec. 4 presents the conclusions and introduces the future work.

\section{Scattering decomposition}

\subsection{Background}

Consider an $(n+1)$-dimensional manifold $\mathcal{D}$, the space of spatial variables, with boundary $\partial \mathcal{D}$ (of dimension $n$ ). The basic assumption is that $\mathcal{D}$ is a Riemannian manifold, i.e. a manifold equipped with a Riemannian metric. To be more precise, two metrics are needed over $\mathcal{D}$ : a volumetric one, generally Euclidean, related to the spatial properties of the domain, and an energetic one, related to the physical phenomena under study inside the domain.

The border $\partial \mathcal{D}$ of the spatial domain $\mathcal{D}$ can be interpreted as the distributed power port on which the interaction between two or more systems takes place. This interaction is simply a power exchange through the boundaries of the interconnected systems.

Intuitively speaking, the difference of 1 in dimension between $\mathcal{D}$ and $\partial \mathcal{D}$ makes it possible to define the internal part of the domain and its external one or, equivalently, a direction along which the power flow can be measured. Since it makes sense to talk about power flow only on the border of the spatial domain (see Sec. 3 for Maxwell's equations and Poynting theorem), the scattering decomposition is defined over there and acts on the restrictions on the border of the power variables.

\subsection{Basic definitions and results}

Throughout the remaining part of this section, assume that $\partial \mathcal{D} \equiv \mathcal{N}$. So, $\mathcal{N}$ is an $n$-dimensional manifold equipped with an embedded Riemannian metric. Moreover, suppose that $\mathcal{N}$ is orientable. Denote by $\Omega^{k}(\mathcal{N})$, $k=0, \ldots, n$, the space of $k$-forms on $\mathcal{N}$, that is the space of $k$-linear alternating functions on $\mathcal{N} ; \Omega^{k}(\mathcal{N})$ is an infinite dimensional linear space with respect to addition and multiplication by elements in $\mathbb{R}$. Moreover, assume $\Omega^{k}(\mathcal{N}) \equiv \Omega^{k}(\partial \mathcal{D})$ as the space of flows on $\partial \mathcal{D}$, [4]. It is possible to identify the dual of $\Omega^{k}(\mathcal{N})$ by means of the following Proposition, proved in [4].

Proposition 2.1. The dual space $\left(\Omega^{k}(\mathcal{N})\right)^{*}$ of $\Omega^{k}(\mathcal{N})$ can be identified with $\Omega^{n-k}(\mathcal{N})$, replacing the duality 
product between $\Omega^{k}(\mathcal{N})$ and $\left(\Omega^{k}(\mathcal{N})\right)^{*}$ by

$$
\langle\beta, \alpha\rangle:=\int_{\mathcal{N}} \beta \wedge \alpha
$$

with $\alpha \in \Omega^{k}(\mathcal{N})$ and $\beta \in \Omega^{n-k}(\mathcal{N})$.

Note 2.1. According to Prop. 2.1, it is possible to identify the space $\mathcal{F}$ of flows in $\mathcal{N}$ with $\Omega^{k}(\mathcal{N})$ and its dual space $\mathcal{E}$ of efforts with $\Omega^{n-k}(\mathcal{N})$. Moreover, (1) provides the definition of dual product.

A key-point in the proof of Prop. 2.1 is the Hodge star operator $\star$. This operator will play an essential role also in the development of the scattering decomposition when the space of power variables is given over a space of forms. From [6], we take the following

Definition 2.1 (Hodge star operator). Consider an $n$-dimensional Riemannian manifold $\mathcal{N}$ with orthonormal and oriented base $v_{1}, \ldots, v_{n}$. The Hodge star operator defines a mapping $\star: \Omega^{k}(\mathcal{N}) \rightarrow \Omega^{n-k}(\mathcal{N})$, such that, given $\beta \in \Omega^{k}(\mathcal{N})$,

$$
(\star \beta)\left(v_{k+1}, \ldots, v_{n}\right):=\beta\left(v_{1}, \ldots, v_{k}\right)
$$

Note 2.2. The definition of Hodge star operator explicitly requires that the manifold $\mathcal{N}$ is equipped with a Riemannian metric $g_{i j}$. Moreover, it is possible to prove, see again [6], that, given $\beta, \beta_{1}, \beta_{2} \in \Omega^{k}(\mathcal{N})$, then the Hodge star operator satisfies the following properties:

$$
\begin{gathered}
\star \star \beta=(-1)^{k(n-k)} \beta \\
\star \beta_{1} \wedge \beta_{2}=\star \beta_{2} \wedge \beta_{1} \\
\int_{\mathcal{N}} \star \beta_{2} \wedge \beta_{1} \text { is an inner product on } \Omega^{k}(\mathcal{N})
\end{gathered}
$$

An important consequence of (2c) is the following

Proposition 2.2. Suppose that $\left(\alpha_{i}, \beta_{i}\right),(\alpha, \beta) \in$ $\Omega^{k}(\mathcal{N}) \times \Omega^{n-k}(\mathcal{N}), i=1,2$. Then

$$
\begin{aligned}
& \left(\left(\alpha_{1}, \beta_{1}\right) \mid\left(\alpha_{2}, \beta_{2}\right)\right):= \\
& \int_{\mathcal{N}} \star \alpha_{2} \wedge \alpha_{1}+(-1)^{k(n-k)} \int_{\mathcal{N}} \beta_{1} \wedge \star \beta_{2}
\end{aligned}
$$

is an inner product on $\Omega^{k}(\mathcal{N}) \times \Omega^{n-k}(\mathcal{N})$, and

$$
\|(\alpha, \beta)\|^{2}:=\int_{\mathcal{N}} \star \alpha \wedge \alpha+(-1)^{k(n-k)} \int_{\mathcal{N}} \beta \wedge \star \beta
$$

is a norm on $\Omega^{k}(\mathcal{N}) \times \Omega^{n-k}(\mathcal{N})$.

Since Prop. 2.1 holds, the following definition of +pairing operator in the infinite dimensional contest can follow.

Definition 2.2 (+pairing opearator). Suppose that $\left(\alpha_{i}, \beta_{i}\right) \in \Omega^{k}(\mathcal{N}) \times \Omega^{n-k}(\mathcal{N}), i=1,2$. Then

$$
\begin{gathered}
\ll\left(\alpha_{1}, \beta_{1}\right),\left(\alpha_{2}, \beta_{2}\right) \gg:=\left\langle\beta_{2}, \alpha_{1}\right\rangle+\left\langle\beta_{1}, \alpha_{2}\right\rangle \\
=\int_{\mathcal{N}}\left(\beta_{2} \wedge \alpha_{1}+\beta_{1} \wedge \alpha_{2}\right)
\end{gathered}
$$

As in $[2,3]$ for the finite dimensional case, the scattering decomposition is possible when a +pairing operator, or a dual product operation, and a metric are defined over the space of power variables. In the finite dimensional case, a metric can be interpreted as a nonsingular linear operator mapping vectors to co-vectors, i.e. flows to efforts. In the classical example of the transmission line, the natural metric is the impedance of the line, that is a physical entity depending on the electromagnetic properties of the line itself (distributed inductance and capacitance), and mapping a current (flow) to a voltage (effort).

Within the framework of infinite dimensional port Hamiltonian systems, the role of the metric is played by the Hodge star operator since it is a map between $k$-forms (flows) to $(n-k)$-forms (efforts). The physical properties of the system are summarized by the Riemannian metric $g_{i j}$ on $\mathcal{N}$, the starting point in the definition of the Hodge operator.

Definition 2.3 (scattering subspaces). Suppose that $\mathcal{N}$ is an $n$-dimensional Riemannian manifold and assume that $\mathcal{F}=\Omega^{k}(\mathcal{N})$ is the space of flows and that $\mathcal{E}=\Omega^{n-k}(\mathcal{N})$ is the space of efforts. The scattering subspaces $\mathcal{S}^{+}, \mathcal{S}^{-} \subset \mathcal{F} \times \mathcal{E}$ are defined as

$$
\begin{aligned}
& \mathcal{S}^{+}:=\left\{(f, e) \mid \ll(f, e),(f, e) \gg=\|(f, e)\|^{2}\right\} \\
& \mathcal{S}^{-}:=\left\{(f, e) \mid \ll(f, e),(f, e) \gg=-\|(f, e)\|^{2}\right\}
\end{aligned}
$$

Note 2.3. From (3b) in Prop. 2.2 and Def. 2.2, we can alternatively write that

$$
\begin{aligned}
\mathcal{S}^{+}= & \left\{(f, e) \in \mathcal{F} \times \mathcal{E} \mid 2 \int_{\mathcal{N}} e \wedge f=\right. \\
& \left.=\int_{\mathcal{N}} \star f \wedge f+(-1)^{k(n-k)} \int_{\mathcal{N}} e \wedge \star e\right\}
\end{aligned}
$$

and

$$
\begin{aligned}
\mathcal{S}^{-}= & \left\{(f, e) \in \mathcal{F} \times \mathcal{E} \mid 2 \int_{\mathcal{N}} e \wedge f=\right. \\
& \left.=-\int_{\mathcal{N}} \star f \wedge f-(-1)^{k(n-k)} \int_{\mathcal{N}} e \wedge \star e\right\}
\end{aligned}
$$

It is important to note the strict relation between scattering subspaces and the Hodge star operator. Since this operator can be defined only if the spatial domain is equipped with a Riemannian metric, this metric is fundamental also for the definition of the scattering subspaces. In Sec. 3, it is shown that it combines both the volumetric and physical properties of the domain.

Proposition 2.3. The scattering subspaces of Def. 2.3 can be written as

$$
\mathcal{S}^{+}=\left\{(f, e) \mid f=(-1)^{k(n-k)} \star e \Leftrightarrow e=\star f\right\}
$$

and

$$
\begin{aligned}
\mathcal{S}^{-}=\{(f, e) \mid e=- & \star f \Leftrightarrow \\
\Leftrightarrow f & \left.=-(-1)^{k(n-k)} \star e\right\}
\end{aligned}
$$


Proof. We start proving that $(4 a) \Rightarrow(5 a)$. Since

$$
2 \int_{\overline{\mathcal{N}}} e \wedge f=\int_{\tilde{\mathcal{N}}} \star f \wedge f+(-1)^{k(n-k)} \int_{\tilde{\mathcal{N}}} e \wedge \star e
$$

for all $\ddot{\mathcal{N}} \subseteq \mathcal{N}$, we deduce that

$$
2 e \wedge f=\star f \wedge f+(-1)^{k(n-k)} e \wedge \star e
$$

or, equivalently, that

$$
(\star f-e) \wedge f+e \wedge\left((-1)^{k(n-k)} \star e-f\right)=0
$$

for all $f \in \mathcal{F}$ and $e \in \mathcal{E}$. This can be true if $e=\star f$ or, equivalently, if $f=(-1)^{k(n-k)} \star e$, as indicated in (5a). Moreover, it is immediate to verify that $(5 a) \Rightarrow(4 a)$. It is only needed to observe that $(6)$ is satisfied if it is assumed that $e=\star f$, or equivalently, that $f=(-1)^{k(n-k)} \star e$.

Applying the same procedure, it is possible to prove that $(4 b) \Leftrightarrow(5 b)$.

Proposition 2.4. These conditions hold

(1) $\mathcal{S}^{+} \cap \mathcal{S}^{-}=\{(0,0)\}$

(2) $\mathcal{F}, \mathcal{E}, \mathcal{S}^{+}$and $\mathcal{S}^{-}$are isomorphic spaces

(3) if $s^{+}=\left(f^{+}, e^{+}\right) \in \mathcal{S}^{+}$and $s^{-}=\left(f^{-}, e^{-}\right) \in \mathcal{S}^{-}$, then $\left(s^{+} \mid s^{-}\right)=0$. This means that $s^{+} \perp s^{-}$with respect to the scalar product on $\mathcal{F} \times \mathcal{E}$ defined by (3a) in Prop. 2.2.

Proof. (1) is a direct consequence of Def. 2.3 while (2) is immediate from Def. 2.1 and Note 2.3. As regard (3), since (2b) holds, we have that

$$
\begin{aligned}
& \left(s^{+} \mid s^{-}\right)=\left(\left(f^{+}, e^{+}\right) \mid\left(f^{-}, e^{-}\right)\right)= \\
& \int_{\mathcal{N}^{-}} \star f^{-} \wedge f^{+}+(-1)^{k(n-k)} \int_{\mathcal{N}} e^{-} \wedge \star e^{+}
\end{aligned}
$$

but $\star f^{-}=-e^{-}$and $(-1)^{k(n-k)} \star e^{+}=f^{+}$, and so

$$
\left(s^{+} \mid s^{-}\right)=-\int_{\mathcal{N}} e^{-} \wedge f^{+} \int_{\mathcal{N}} e^{-\wedge f^{+}}=0
$$

Note 2.4. Consequence of the previous proposition is that $\mathcal{F} \times \mathcal{E}=\mathcal{S}^{+} \oplus \mathcal{S}^{-}$, with $\mathcal{S}^{+}$and $\mathcal{S}^{-}$orthogonal in the sense of the inner product (3a).

Proposition 2.5. The tpairing restricted on $\mathcal{S}^{+}$or on $\mathcal{S}^{-}$gives the inner product $(\cdot \mid \cdot)$.

Proof. If $s_{i}^{+}=\left(f_{i}^{+}, e_{i}^{+}\right) \in \mathcal{S}^{+}, i=1,2$, then

$$
\begin{aligned}
\ll s_{1}^{+}, & s_{2}^{+} \gg=\int_{\mathcal{N}}\left(e_{2}^{+} \wedge f_{1}^{+}+e_{1}^{+} \wedge f_{2}^{+}\right) \\
= & \int_{\mathcal{N}} \star f_{1}^{+} \wedge f_{2}^{+}+(-1)^{k(n-k)} \int_{\mathcal{N}} e_{2}^{+} \wedge \star e_{1}^{+} \\
& =\left(\left(f_{1}^{+}, e_{1}^{+}\right) \mid\left(f_{2}^{+}, e_{2}^{+}\right)\right)=\left(s_{1}^{+} \mid s_{2}^{+}\right)
\end{aligned}
$$

In the same way if $s_{i}^{-}=\left(f_{i}^{-}, e_{i}^{-}\right) \in \mathcal{S}^{-}, i=1,2$.
Note 2.5. Applying the same procedure as in the previous proposition, it is also possible to prove that, if $s^{+}=\left(f^{+}, e^{+}\right) \in \mathcal{S}^{+}$and $s^{-}=\left(f^{-}, e^{-}\right) \in \mathcal{S}^{-}$, then $\ll s^{+}, s^{-} \gg=0$.

Before stating the infinite dimensional version of the Scattering Power Decomposition Theorem, $[2,3]$, it is important to note that, given $(f, e) \in \mathcal{F} \times \mathcal{E}$, are unequivocally determined $s^{+}=\left(f^{+}, e^{+}\right) \in \mathcal{S}^{+}$and $s^{-}=$ $\left(f^{-}, e^{-}\right) \in \mathcal{S}^{-}$such that $(f, e)=\left(f^{+}+f^{-}, e^{+}+e^{-}\right)=$ $s^{+}+s^{-}$.

Theorem 2.6 (scattering power decomposition). Given $(f, e) \in \mathcal{F} \times \mathcal{E}$, the following relation holds:

$$
\langle e, f\rangle=\frac{1}{2}\left\|s^{+}\right\|^{2}-\frac{1}{2}\left\|s^{-}\right\|^{2}
$$

where $s^{+}=\left(f^{+}, e^{+}\right) \in \mathcal{S}^{+}, s^{-}=\left(f^{-}, e^{-}\right) \in \mathcal{S}^{-}$, $(f, e)=s^{+}+s^{-}$and the metric $\|\cdot\|^{2}$ is the one defined by (3b) and restricted on $\mathcal{S}^{+}$and $\mathcal{S}^{-}$.

Proof. From Prop. 2.5 and Note 2.5, we have that

$$
\begin{aligned}
& 2\langle e, f\rangle=\ll(f, e),(f, e) \gg= \\
& =\ll\left(f^{+}, e^{+}\right),\left(f^{+}, e^{+}\right) \gg+\ll\left(f^{-}, e^{-}\right),\left(f^{-}, e^{-}\right) \gg \\
& =\left\|\left(f^{+}, e^{+}\right)\right\|^{2}-\left\|\left(f^{-}, e^{-}\right)\right\|^{2}=\left\|s^{+}\right\|^{2}-\left\|s^{-}\right\|^{2}
\end{aligned}
$$

and finally

$$
\langle e, f\rangle=\frac{1}{2}\left\|s^{+}\right\|^{2}-\frac{1}{2}\left\|s^{-}\right\|^{2}
$$

Note 2.6. The exchanged power between the system and the environment through its boundary can be written as the sum of a positive and a negative power flow. The scattering variable $s^{+}$could be related to an incoming power flow and $s^{-}$to an outgoing power flow. This decomposition is always possible if a certain metric operator is defined on the space of power variables. As already pointed out, this metric is strictly related to the physical properties of the systems. Different properties lead to different scattering decompositions.

\subsection{The scattering mapping}

The scattering mapping is a map between the space of power variables $\mathcal{F} \times \mathcal{E}$ and the space of scattering variables $\mathcal{S}^{+} \oplus \mathcal{S}^{-}$. It is possible to extend the results for the finite dimensional case, see again $[2,3]$, to the case of infinite dimensional systems.

As in the finite dimensional situation, the first step is to find a proper coordinate representation for both the space of power variables and the space of scattering variables. Since $\mathcal{F}=\Omega^{k}(\mathcal{N})$, if $x_{1}, \ldots, x_{n}$ is a set of local coordinates on $\mathcal{N}$, then each flow $f \in \mathcal{F}$ can be written as a linear combination of simple $k$-forms

$$
d x^{I}:=d x^{i_{1}} \wedge \cdots \wedge d x^{i_{k}}
$$


with $I=\left\{i_{1}, \ldots, i_{k}\right\}$ a multi-index of order $k$ and $1 \leq$ $i_{1}<\cdots<i_{k} \leq n$. So, we can state that, given $f \in \mathcal{F}$, a set of functions $f_{I}: \mathcal{N} \rightarrow \mathbb{R}$ such that $f=f_{I} d x^{I}$ is unequivocally determined.

In the same way, a base for $\mathcal{E}=\Omega^{n-k}(\mathcal{N})$ is given by the set of all

$$
d x^{J}=d x^{j_{1}} \wedge \cdots \wedge d x^{j_{n-k}}
$$

with $J=\left\{j_{1}, \ldots, j_{n-k}\right\}$ a multi-index of order $n-k$ and $1 \leq j_{1}<\cdots<j_{n-k} \leq n$, and, for every $e \in \mathcal{E}$, a set of $e_{J}: \mathcal{N} \rightarrow \mathbb{R}$ such that $e=e_{J} d x^{J}$ is unequivocally determined.

It is also possible to define a base for each scattering subspace $\mathcal{S}^{+}$and $\mathcal{S}^{-}$, with the characteristic of being orthogonal in the sense of $(\cdot \mid \cdot)$. This can be done by means of the following

Proposition 2.7. The sets

$$
\left\{s^{+, I}:=\frac{\sqrt{2}}{2}\left(d x^{I}, \star d x^{I}\right) \in \mathcal{S}^{+}\right\}
$$

and

$$
\left\{s^{-, I}:=\frac{\sqrt{2}}{2}\left(-d x^{I}, \star d x^{I}\right) \in \mathcal{S}^{-}\right\}
$$

with $I=\left\{i_{1}, \ldots, i_{k}\right\}, 1 \leq i_{1}<\cdots<i_{k} \leq n$, give an orthogonal base of $\mathcal{S}^{+}$and $\mathcal{S}^{-}$in the sense of $(\cdot \mid \cdot)$ on $\mathcal{F} \times \mathcal{E}$. This is what we call flow-representation of the scattering subspaces. Moreover, also the sets

$$
\left\{s^{+, J}:=\frac{\sqrt{2}}{2}\left((-1)^{k(n-k)} \star d x^{J} ; d x^{J}\right) \in \mathcal{S}^{+}\right\}
$$

and

$$
\left\{s^{-, J}:=\frac{\sqrt{2}}{2}\left(-(-1)^{k(n-k)} \star d x^{J}, d x^{J}\right) \in \mathcal{S}^{-}\right\}
$$

with $J=\left\{j_{1}, \ldots, j_{n-k}\right\}, 1 \leq j_{1}<\cdots<j_{n-k} \leq n$, give an orthogonal base of $\mathcal{S}^{+}$and $\mathcal{S}^{-}$in the sense of $(\cdot \mid \cdot)$. We call this the effort-representation of the scattering subspaces.

Proof. Only the proof for the orthogonality of the bases in the flow-representation (8) is given. Let start with the set defined in (8a). Consider $s^{+, I_{1}}, s^{+, I_{2}} \in \mathcal{S}^{+}$. With some simple calculations we obtain that

$$
\left(s^{+, I_{1}} \mid s^{+, I_{2}}\right)=\int_{\mathcal{N}} \star d x^{I_{2}} \wedge d x^{I_{1}}
$$

which is 0 if and only if $I_{1} \neq I_{2}$. The same procedure can be applied to prove that the set of $s^{-, I}$, defined in (8b), are an orthogonal base for $\mathcal{S}^{-}$. If $s^{-, I_{1}}, s^{-, I_{2}} \in$ $\mathcal{S}^{-}$, then again

$$
\left(s^{-, I_{1}} \mid s^{-: I_{2}}\right)=\int_{\mathcal{N}} \star d x^{I_{2}} \wedge d x^{I_{1}}
$$

which is 0 if and only if $I_{1} \neq I_{2}$.
Consider two multi-index $I$ and $J$, with $I=$ $\left\{i_{1}, \ldots, i_{k}\right\}, 1 \leq i_{1}<\cdots<i_{k} \leq n$, and $J=$ $\left\{j_{1}, \ldots, j_{n-k}\right\}, 1 \leq j_{1}<\cdots<j_{n-k} \leq n$. If $g_{i j}$ is the Riemannian metric on $\mathcal{N}$, from Def. 2.1, it is possible to prove that (see [6])

$\star d x^{I}=\sqrt{g} \varepsilon_{l_{1} \ldots l_{k} j_{1} \ldots j_{n-k}} g^{i_{1} l_{1}} \ldots g^{i_{k} l_{k}} d x^{j_{1}} \wedge \cdots \wedge d x^{j_{n-k}}$ where $g=\operatorname{det}\left[g_{i j}\right], \varepsilon$ is the Levi-Civita tensor and the sum is extended to all $l_{1}, \ldots, l_{k}$, with $1 \leq l_{1}<\cdots<$ $l_{k} \leq n$, and to all $j_{1}, \ldots, j_{n-k}$, with $1 \leq j_{1}<\cdots<$ $j_{n-k} \leq n$. Moreover,

$\star d x^{J}=\sqrt{g} \varepsilon_{l_{1} \ldots l_{n-k} i_{1} \ldots i_{k}} g^{i_{1} l_{1}} \ldots g^{i_{n-k} l_{n-k}} d x^{i_{1}} \wedge \cdots \wedge d x^{i_{k}}$

where the sum is extended to all $l_{1}, \ldots, l_{n-k}$, with $1 \leq$ $l_{1}<\cdots<l_{n-k} \leq n$, and to all $i_{1}, \ldots, i_{k}$, with $1 \leq i_{1}<$ $\cdots<i_{k} \leq n$. In a compact notation, we can say that

$$
\begin{aligned}
\star d x^{I} & =\sqrt{g} \varepsilon_{L, \bar{J}} g^{I, L} d x^{\tilde{J}} \\
\star d x^{J} & =\sqrt{g} \varepsilon_{L, \bar{I}} g^{J, L} d x^{\bar{I}}
\end{aligned}
$$

Consider $(f, e) \in \mathcal{F} \times \mathcal{E}$. We know that a set of functions $f_{I}, e_{J}: \mathcal{N} \rightarrow \mathbb{R}$ such that $f=f_{I} d x^{I}$ and $e=e_{J} d x^{J}$ are unequivocally determined. Clearly, if it is assumed a flow-representation for the scattering subspaces, with

$$
\begin{aligned}
& s^{+, I}=\frac{\sqrt{2}}{2}\left(d x^{I}, \star d x^{I}\right) \\
& s^{-, I}=\frac{\sqrt{2}}{2}\left(-d x^{I}, \star d x^{I}\right)
\end{aligned}
$$

as in Prop. 2.7, then, from Prop. 2.4, we deduce that also a set of functions $s_{I}^{+}, s_{I}^{-}: \mathcal{N} \rightarrow \mathbb{R}$ such that

$$
(f, e)=s_{I}^{+} s^{+, I}+s_{I}^{-} s^{-, I}
$$

is unequivocally determined. This observation leads to the following

Proposition 2.8 (scattering mapping). Suppose that a base for $\mathcal{S}^{+}$and $\mathcal{S}^{-}$is chosen according to the flow-representation (8) in Prop. 2.7 and consider $(f, e) \in \mathcal{F} \times \mathcal{E}$, with $f=\sum_{I} f_{I} d x^{I}$ and $e=\sum_{J} e_{J} d x^{J}$ in coordinates. If $s^{+} \in \mathcal{S}^{+}$and $s^{-} \in \mathcal{S}^{-}$are the corresponding scattering variables, expressed as $s^{+}=$ $\sum_{I} s_{I}^{+} s^{+, I}$ and $s^{-}=\sum_{I} s_{I}^{-} s^{-, I}$ in coordinates, then

$$
\left\{\begin{array}{l}
f_{I}=\frac{\sqrt{2}}{2}\left(s_{I}^{+}-s_{I}^{-}\right) \\
e_{J}=\frac{\sqrt{2}}{2} \sqrt{g} \varepsilon_{L, J} g^{\bar{I}, L}\left(s_{\bar{I}}^{+}+s_{\bar{I}}^{-}\right)
\end{array}\right.
$$

or, equivalently,

$$
\left\{\begin{array}{l}
s_{I}^{+}=\frac{\sqrt{2}}{2}\left((-1)^{k(n-k)} \sqrt{g} \varepsilon_{L, I} g^{\bar{J}, L} e_{j}+f_{I}\right) \\
s_{I}^{-}=\frac{\sqrt{2}}{2}\left((-1)^{k(n-k)} \sqrt{g} \varepsilon_{L, I} g^{\tilde{J}, L} e_{\tilde{J}}-f_{I}\right)
\end{array}\right.
$$

for every multi-index $I=\left\{i_{1}, \ldots, i_{k}\right\}, 1 \leq i_{1}<\cdots<$ $i_{k} \leq n$. 
Proof. From (11) we have that

$$
f=f_{I} d x^{I}=\frac{\sqrt{2}}{2}\left(s_{I}^{+}-s_{I}^{-}\right) d x^{I}
$$

and

$$
e=e_{J} d x^{J}=\frac{\sqrt{2}}{2} \sqrt{g} \varepsilon_{L, J} g^{\tilde{I}, L}\left(s_{\bar{I}}^{+}+s_{\tilde{I}}^{-}\right) d x^{J}
$$

that gives (12a). The inverse relations $(12 \mathrm{~b})$ can be obtained as follows. First of all, from the second of (12a) we have

$$
\begin{aligned}
& (-1)^{k(n-k)} \sqrt{g} \varepsilon_{M, I} g^{\tilde{J}, M} e_{\tilde{J}}= \\
& =\frac{\sqrt{2}}{2}(-1)^{k(n-k)} g \varepsilon_{M, I} \varepsilon_{L, \tilde{J}} g^{\tilde{J}, M} g^{\bar{I}, L}\left(s_{\tilde{I}}^{+}-\overrightarrow{s_{\tilde{I}}}\right)
\end{aligned}
$$

Since

$$
(-1)^{k(n-k)} \varepsilon_{M, I} \varepsilon_{L, j} g^{\tilde{J}, M} g^{\tilde{I}, L}=\frac{1}{g} \delta_{I}^{\tilde{I}}
$$

with $\delta$ the Kronecker delta, we obtain

$$
\frac{\sqrt{2}}{2}\left(s_{I}^{+}+s_{I}^{-}\right)=(-1)^{k(n-k)} \sqrt{g} \varepsilon_{M, I} g^{\bar{J}, M} e_{\tilde{J}}
$$

that, if summed with the first relation in (12a) gives the first in (12b)

$$
f_{I}+(-1)^{k(n-k)} \sqrt{g} \varepsilon_{M, I} g^{\bar{J}, M} e_{j}=\sqrt{2} s_{I}^{+}
$$

and, if subtracted, gives the second in (12b)

$$
f_{I}-(-1)^{k(n-k)} \sqrt{g} \varepsilon_{M, I} g^{j, M} e_{\tilde{J}}=-\sqrt{2} s_{I}^{-}
$$

\section{Example: Maxwell's equations}

Consider some connected and closed domain $\mathcal{D}$ of the three-dimensional oriented Euclidean space $\mathbb{E}^{3}$, or, equivalently, suppose that $\mathcal{D}$ is a three-dimensional close and connected Riemannian manifold equipped with the Euclidean metric $\delta_{i j}$, being $\delta$ the Kronecker delta. Moreover, assume that in $\mathcal{D}$ some electromagnetic phenomena, described by the Maxwell's equations, are taking place. Following the formulation of Maxwell's equations in terms of differential forms [4, 7], the co-energy variables are the 1 -forms electric field intensity $E$ and the magnetic field intensity $H$, with $E, H \in \Omega^{1}(\mathcal{D})$. Moreover, the energy variables are the 2 -forms electric field induction $D$ and the magnetic field induction $B$, with $D, B \in \Omega^{2}(\mathcal{D})$.

If the medium is linear and non-bianisotropic [9], its macroscopic electric and magnetic properties can be described by the following $(0,2)$ symmetric and positive definite tensors, the electric permittivity $\epsilon_{i j}$ and the magnetic permittivity $\mu_{i j}$. It is correct to assume that

$$
\epsilon_{i j}=\epsilon_{i}^{k} g_{i j}=\epsilon_{i}^{k} \delta_{k j} \quad \mu_{i j}=\mu_{i}^{k} g_{i j}=\mu_{i}^{k} \delta_{k j}
$$

where $g_{i j}=\delta_{i j}$ is the (spatial) Euclidean metric on $\mathcal{D}$. We have that $\epsilon_{i}^{j}$ and $\mu_{i}^{j}$ are linear operator, symmetric with respect to the metric defined on $\mathcal{D}$. Moreover, $\epsilon_{i j}$ and $\mu_{i j}$ can be treated as positive definite metrics on $\mathcal{D}$ and, consequently, used to define two distinct Hodge star operators $\star_{\epsilon}$ and $\star_{\mu}$. These Hodge operators are not needed to define the scattering decomposition on the border $\partial \mathcal{D}$ of the physical domain, but only to write the constitutive relations of the medium and the electromagnetic energy density. In particular, the constitutive relations, that is the relations between energy and co-energy variables, are given by

$$
D=\star_{\epsilon} E \quad B=\star_{\mu} H
$$

and the electromagnetic energy density, a 3-form, by

$$
H_{e m}=\frac{1}{2}(E \wedge D+H \wedge B)=\frac{1}{2}\left(E \wedge \star_{\epsilon} E+H \wedge \star_{\mu} H\right)
$$

If $\mathcal{H}_{e m}=\int_{\mathcal{D}} H_{e m}$ is the total electromagnetic energy, it is well-known that

$$
\frac{d \mathcal{H}_{e m}}{d t}=\int_{\partial \mathcal{D}} E \wedge H
$$

or, equivalently, that the time derivative of the total electromagnetic energy in $\mathcal{D}$ is equal to the flow of electromagnetic power radiating through the boundary $\partial \mathcal{D}$. Locally, the power through the boundary is given by the 2-form $S=E \wedge H \in \Omega^{2}(\partial \mathcal{D})$, the Poynting vector.

Clearly, (15) provides the expression of the duality product for Maxwell's equations on the boundary of the spatial domain. The power conjugated variables are the electric field $E$ restricted on the boundary, that is $E l_{\partial \mathcal{D}}$, and the magnetic field $H$ restricted on the boundary, that is $\left.H\right|_{\partial \mathcal{D}}$. The flow variable is $\left.E\right|_{\partial \mathcal{D}}$ and the effort variable is $\left.H\right|_{\partial \mathcal{D}}$.

In order to define a scattering decomposition over the boundary of the spatial domain, a dual product and a metric, that is a 1-1 mapping between flow and effort variables, are needed (see Def. 2.3). Since (15) provides the definition of the duality product, the next step is to find a proper metric on the space of power variables.

Given the Euclidean metric $\delta_{i j}$ and the electric permittivity and magnetic permeability tensors $\epsilon_{i j}$ and $\mu_{i j}$ on $\mathcal{D}$, it is possible to consider their restrictions $g_{i^{\prime} j^{\prime}}, \epsilon_{i^{\prime} j^{\prime}}$ and $\mu_{i^{\prime} j^{\prime}}$ on $\partial \mathcal{D}$, [8]. All these tensors are symmetric and positive definite, so it is possible to assume that

$$
\epsilon_{i^{\prime} j^{\prime}}=\epsilon_{i^{\prime}}^{k^{\prime}} g_{k^{\prime} j^{\prime}} \quad \mu_{i^{\prime} j^{\prime}}=\mu_{i^{\prime}}^{k^{\prime}} g_{k^{\prime} j^{\prime}}
$$

where $\epsilon_{i^{\prime}}^{j^{\prime}}$ and $\mu_{i^{\prime}}^{j^{\prime}}$ are linear operators on $\partial \mathcal{D}$, that are also symmetric and positive definite with respect to the induced metric $g_{i^{\prime} j^{\prime}}$ on $\partial \mathcal{D}$. They can be interpreted as the restrictions of the linear operators $\epsilon_{j}^{i}$ and $\mu_{j}^{i}$, introduced in (14), on $\partial \mathcal{D}$. If $\epsilon_{j^{\prime}}^{i^{\prime}}=(\sqrt{\epsilon})_{k^{\prime}}^{i^{\prime}}(\sqrt{\epsilon})_{j^{\prime}}^{k^{\prime}}$ and 
$\mu_{j^{\prime}}^{i^{\prime}}=(\sqrt{\mu})_{k^{\prime}}^{i^{\prime}}(\sqrt{\mu})_{j^{\prime}}^{k^{\prime}}$, with $(\sqrt{\epsilon})_{j^{\prime}}^{i^{\prime}}$ and $(\sqrt{\mu})_{j^{\prime}}^{i^{\prime}}$ symmetric and positive definite linear operators, with respect to the metric on $\partial \mathcal{D}$, it is possible to define the following metric on $\partial \mathcal{D}$ :

$$
z_{i^{\prime} j^{\prime}}:=(\sqrt{\epsilon})_{i^{\prime}}^{k^{\prime}}\left(\sqrt{\mu^{-1}}\right)_{k^{\prime}}^{l^{\prime}} g l^{\prime} j^{\prime}
$$

where $g_{i^{\prime} j^{\prime}}$ is the restriction of the Euclidean metric on $\partial \mathcal{D}$ and $\left(\sqrt{\mu^{-1}}\right)_{j^{\prime}}^{i^{\prime}}$ is the inverse (linear) mapping of $(\sqrt{\mu})_{j^{\prime}}^{i^{\prime}}$. Obviously, $\left(\sqrt{\mu^{-1}}\right)_{j^{\prime}}^{i^{\prime}}$ is symmetric and positive definite respect to $g_{i^{\prime} j^{\prime}}$.

If $\star_{z}$ is the Hodge star operator defined using $z_{i^{\prime} j^{\prime}}$ as metric on $\partial \mathcal{D}$, then it defines a 1-1 mapping between the space of flows and efforts on $\partial \mathcal{D}$. In fact, it is possible to prove that, given the restriction on the border of $\mathcal{D}$ of an electric field $E$, then $H=\star_{z} E$ is the corresponding restriction on $\partial \mathcal{D}$ of a magnetic field. In other words, $\star_{z}$ is the metric required in the definition of the scattering subspaces $\mathcal{S}^{+}$and $\mathcal{S}^{-}$on the boundary of the physical domain $\mathcal{D}$ (see Def 2.3). The assumption (16) for the metric on $\partial \mathcal{D}$ can be intuitively justified as follows: since the electromagnetic energy can be written as $\frac{1}{2}\left(\epsilon E^{2}+\mu H^{2}\right)=\frac{1}{2} \mu\left(\epsilon / \mu E^{2}+H^{2}\right)$, then, in some sense, we have that $\sqrt{\epsilon / \mu} E$ is equivalent to a magnetic field $H$. At this point, all the results described in Sec. 2 can be obtained by simple calculations.

In conclusion, the example of Maxwell's equations points out that the physical properties of an infinite dimensional systems can be summarized by a spatial metric and two linear operators, symmetric and positive definite with respect to the spatial metric (e.g. $\delta_{i j}, \epsilon_{j}^{i}$ and $\mu_{j}^{i}$ ). Inside the domain, these tensors are combined in such a way that two Hodge operators, $\star_{\epsilon}$ and $\star_{\mu}$, can be defined. By means of these operators, the constitutive relations and the energy density can be written. On the border of the domain, a proper combination of the restriction of the same tensors gives a Riemannian metric $z_{i^{\prime} j^{\prime}}$ and, consequently, another Hodge operator $\star_{z}$. Then, $\star_{z}$ is a metric on the space of power variables or, equivalently, a mapping between flows and efforts. In other words, it is the impedance in the infinite dimensional case. These different combinations are summarized in Fig. 1.

\section{Conclusions and future work}

In this paper, the generalization of impedance for infinite dimensional systems within the framework of port Hamiltonian systems is presented in order to extend the scattering decomposition to the infinite dimensional case and treat generic power propagation phenomena. The results of Sec. 3 for Maxwell's equation can be easily extended, for example, to flexible structures. In particular, an analogous of Maxwell's equations can be

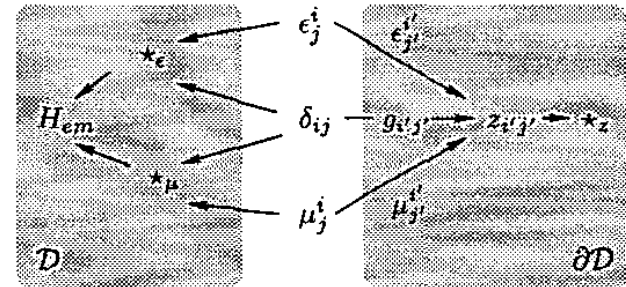

Figure 1: Different combinations of the spatial metric $\delta_{i j}$ and of the linear operators $\epsilon_{j}^{i}$ and $\mu_{j}^{i}$ in $\mathcal{D}$ and on its border $\partial \mathcal{D} ; \star_{z}$ can be considered as the impedance in the infinite dimensional case.

obtained to describe the behavior of (simple) flexible beams. The analysis of more complex models of flexible structures and the study of the interconnection of infinite dimensional systems in terms of scattering will be the main topics of our future work.

Acknowledgments. This work has been done in the context of the European sponsored project Geoplex with reference code IST-2001-34166. Further information is available at http://www. geoplex.cc.

\section{References}

[1] Lax P.D. and R.S. Phillips, Scattering Theory, Pure and Applied Mathematics, Academic Press, New York, 1967.

[2] Stramigioli S., A.J. van der Schaft, B. Maschke and C. Melchiorri, Geometric Scattering in Robotic Telemanipulation, submitted to the IEEE Transactions of Robotics and Automation, 2001.

[3] Stramigioli, S., Modeling and Ipc Control of Interactive Mechanical Systems. A Coordinate - Free Approach, Lecture Notes in Control and Information Sciences, Springer-Verlag, 2001.

[4] Maschke, B.M.J. and A.J. van der Schaft, Port controlled Hamiltonian representation of distributed parameter systems, 2001.

[5] van der Schaft, A.J., $L_{2}$-gain and passivity techniques in nonlinear control, Springer-Verlag London, 2000.

[6] Abraham, R. and J.E. Marsden, Foundation of Mechanics, Benjamin/Cummings Publishing Company, 2nd edition, 1978

[7] Ingarden, R.S. and Jamiolkowski, Classical Electrodynamics, PWN-Polish Sc. Publ., Warszawa, Elsevier, 1985.

[8] Dubrovin B.A., A.T. Fomenko and S.P. Novikov, Modern Geometry - Methods and Application (Part I), Springer-Verlag New York, 1992.

[9] Warnick, K.F. and D.V. Arnold, Green Form for Anisotropic, Inhomogeneous Media, 1997. 